\title{
Genetic Programming for Experimental Big Data Mining: A Case Study on Concrete Creep Formulation
}

\author{
Amir H. Gandomi \\ Distinguished Research Fellow, BEACON Center for the Study of Evolution in Action, Michigan State \\ University, East Lansing, MI 48824, USA. \\ Siavash Sajedi* \\ Research Assistant, Department of Civil Engineering, The University of Akron, Akron, OH 44325, \\ USA. \\ Behnam Kiani \\ Research Assistant, Department of Civil Engineering, The University of Akron, Akron, OH 44325, \\ USA. \\ Qindan Huang \\ Assistant Professor, Department of Civil Engineering, The University of Akron, ASEC 210, Akron, \\ OH 44325, USA.
}

\begin{abstract}
This paper proposes a new algorithm called multi-objective genetic programming (MOGP) for complex civil engineering systems. The proposed technique effectively combines the model structure selection ability of a standard genetic programming with the parameter estimation power of classical regression, and it simultaneously optimizes both the complexity and goodness-of-fit in a system through a nondominated sorting algorithm. The performance of MOGP is illustrated by modeling a complex civil engineering problem: the time-dependent total creep of concrete. A Big Data is used for the model development so that the proposed concrete creep model-referred to as a "genetic programming based creep" or "G-C model" in this study - is valid for both normal and high strength concrete with a wide range of structural properties. The G-C model is then compared with currently accepted creep prediction models. The G-C model obtained by MOGP is simple, straightforward to use, and provides more accurate predictions than other prediction models.
\end{abstract}

Keywords: Multi-gene genetic programming, Big Data, multi-objective optimization, non-dominated sorting, concrete creep

\section{Introduction}

Different techniques can be used for modeling nonlinear systems in structural engineering, and the models obtained from these techniques can be broadly categorized into two groups: phenomenological (or knowledge-based) and behavioral. Phenomenological models consider the physical laws governing the system (such as energy, momentum, etc.). In these models, the structure of the system should be selected by the model developer based on the physical laws, which requires prior knowledge about the system. Due to the complexity of many structural engineering systems/phenomena (such as modeling of

*Corresponding author (Email: ss287@uakron.edu). Tel: +1 3308089965. 
concrete shrinkage and creep), it is not always possible to derive such models. In contrast to phenomenological models, behavioral models can be easily developed by finding the relationships between input variables and outputs for a set of experimental data without considering the physical theories. For developing behavioral models, no prior knowledge is needed about the mechanism or fundamental theory that produced the experimental data. Therefore, behavioral modeling techniques can be used for approximate modeling of many structural engineering systems [1,2].

While behavioral models can be advantageous, many behavioral models require the user to prespecify/hypothesize the formulation structure of the model. In other words, behavioral techniques optimize the unknown coefficients of a pre-defined formulation structure. In particular, regression analysis is a commonly used technique for developing behavioral models. Although this technique can be used for developing both linear and nonlinear models, it has a strong sensitivity to outliers and can exhibit large model errors due to the idealization of complex processes, approximation, and averaging widely varying prototype conditions [3, 4]. Furthermore, for linear regressions, the least square estimate of unknown parameters can be obtained analytically, while nonlinear regressions typically use an iterative optimization procedure to estimate the unknown parameters, which requires the user to provide starting values. Failure in defining the appropriate starting values can lead to convergence problems or finding the local minimum rather than a global minimum in the optimization process. Therefore, using traditional techniques such as regression analysis cannot guarantee that a reliable and accurate behavioral model will be obtained, particularly for complex nonlinear engineering systems.

In recent years, more advanced computer-aided pattern-recognition and data-classification techniques, such as artificial neural networks (ANNs) and support vector machines (SVMs), have been used to develop behavioral models in various civil engineering problems (e.g., [5-8]). ANN discovers patterns and approximates relationships in data based on a supervised learning algorithm, a form of regression that relies on the inputs and outputs of a training set [9]. Although ANNs are generally successful in prediction, they are only appropriate to use as part of a computer program, not for the development of practical prediction equations. In addition, ANN requires data to be initially normalized based on the suitable activation function and the best network architecture to be determined by the user, and it can have a complex structure and a high potential for over-fitting [10]. SVMs, on the other hand, are one of the efficient kernel-based methods that can solve a convex constrained quadratic programming (CCQP) problem to find a set of parameters. However, selecting the appropriate kernel in SVM can be a challenge, and the results are not transparent [11].

One powerful technique for developing nonlinear behavioral models in the case of complex optimization problems is genetic programming (GP) [12]. GP is specialization subset of genetic algorithms (GAs) [13], which are based on the principles of genetics and natural selection. GP and its variants have been successfully used for solving a number of different civil engineering problems (e.g., $[14,15])$. Multi-gene genetic programming (MGGP) is a robust variant of GP that combines the ability of the standard GP in constructing the model structure with the capability of traditional regression in parameter estimation. In this technique, each symbolic model (and each member of the GP population) is a weighted linear combination of low order non-linear transformations of the input variables. In contrast to standard symbolic regression, MGGP allows the evolution of accurate and relatively compact 
mathematical models. Even when large numbers of input variables are used, this technique can automatically select the most contributed variables in the model, formulate the structure of the model, and solve the coefficients in the regression equation [16-19]. Therefore, unlike other techniques such as traditional regression analysis or ANN, there is no need in the MGGP technique for the user to pre-define the formulation structure of the model or select any existing form of the relationship for optimization [3, 4], which makes it more practical for complex optimization problems. Recent studies also show that compared to other novel computer-based techniques such as SVM and particle swarm model selection, GP shows better performance in problems having high dimensionality and large training sets [20].

Typically, standard GP algorithms (including MGGP) will optimize only one objective in the model development process: maximizing the goodness-of-fit to the training data. The main drawback of using a single objective in the optimization process is that the developed models can become overly complex. In other words, minimizing the complexity of the developed models should be another important objective to be considered. In this study, a new algorithm called multi-objective genetic programming (MOGP) is developed. MOGP is an extension of standard GP algorithms that can simultaneously solve for two competing objectives (i.e. maximizing the goodness-of-fit and minimizing the model complexity). By performing multi-gene symbolic regression via MOGP, one can develop parsimonious and accurate data-based models for complex engineering systems.

This paper presents the feasibility of using MOGP for modeling complex nonlinear civil engineering systems. Two objectives are considered for optimization through MOGP: 1) maximization of goodness-of-fit and 2) minimization of model complexity. As an illustration, the capability of this technique is demonstrated by developing a simple and accurate model (referred to as the "genetic programming based creep model" or "G-C model" in this study) for predicting a complex civil engineering phenomenon: the time-dependent total creep compliance of concrete [21]. A large experimental database selected from Northwestern University's Infrastructure Technology Institute (NU-ITI) database [22] is used for the G-C model development; as such, the proposed model is valid for a wide range of structural properties. The multiple imputation method [23] is used to deal with missing data so that the collected data can be incorporated in the model development as much as possible. The predictors are selected from the literature and consist of parameters that have been found to have an influence on the total creep of concrete (such as relative humidity, curing period, etc.). In this study, some schemes have been also used to handle Big Data in genetic programming. The model selection procedure is automatically conducted by MOGP to select the most statistically contributed predictors to obtain an accurate, unbiased, and parsimonious model. To evaluate the capability of the G-C model, its accuracy is compared with other developed models in terms of variant statistical indicators.

\section{Methodology}

\subsection{Multi-gene symbolic regression}

Genetic algorithm (GA) [13] and genetic programming (GP) [12] are two specific types of evolutionary algorithms that have been used in wide range of practical problems in different fields such as 
optimizing a fixed set of variables or finding a global optimum solution [24]. GA is a traditional optimization technique that uses a fixed length linear representation with binary encoding of all parameters; thus, the output of the GA is a string of numbers. Compared with the GA approach, GP solves the optimization problems without requiring the user to know or pre-define the structure of the solution. GP randomly creates a population of possible solutions composed of functions and terminals appropriate to the problem domain for the first generation. New generations are created by genetic operations such as crossover and mutation. Crossover creates a new individual from two parental individuals during the evolutionary process by randomly selecting a node from the parental individuals and exchanging the subtrees under the selected nodes. Mutation creates a new individual from an existing tree in a population by truncating and replacing one node of a tree with another randomly generated node from the same set. The individual with higher fitness values will have a higher probability of surviving in the successive generation. In order to find the best fitting solution, GP modifies the individual solution of a population after a number of runs in each generation and selects the parental individuals from the population based on a suitable fitness function.

Symbolic regression technique can be implemented using standard GP to evolve a population of genes, in which each of the genes directly encodes a symbolic mathematical expression. A typical multi-gene model for this technique, which is known as multi-gene symbolic regression, is shown in Fig. 1. As can be seen in this figure, the response is predicted using three input variables $\left(x_{1}, x_{2}\right.$, and $x_{3}$ ). Although the structure of this model contains nonlinear terms such as "sin" and "log", the overall model is a weighted linear combination of each gene with respect to the coefficients $d_{0}, d_{1}$, and $d_{2}$. The general formulation of the multi-gene symbolic regression model can be expressed as follows:

$\hat{y}(\mathbf{x}, \mathbf{d}, \boldsymbol{\theta})=d_{0}+\sum_{i=1}^{i=n} d_{i} \cdot G_{i}(\boldsymbol{\theta}, \mathbf{x})$

where $d_{0}$ is a bias term, $d_{i}$ is the gene weight, $G_{i}(\boldsymbol{\theta}, \mathbf{x})$ is the vector of outputs from the $i^{\text {th }}$ gene comprising a multi-gene individual, $\boldsymbol{\theta}$ is the vector of the unknown parameters for each gene, and $n$ is the number of genes. Note that the algorithm of MGGP is similar to a standard GP, except for crossover and mutation of multigene individuals. MGGP does not require simplifying assumptions in developing the models, and it is more accurate and efficient than the standard GP for modeling nonlinear complex problems [3, 4]. In MGGP, the initial population is constructed by creating random individuals using different nonlinear functions, input variables, and a range of random constants. Each individual contains between 1 to $G_{\max }$ genes. The algorithm attempts to maximize diversity by ensuring that no individuals contain duplicate genes. The genes are randomly chosen, and the vector of unknown coefficients $\mathbf{d}$ is estimated using least squares normal equation as follows [16-19]:

$\mathbf{d}=\left(\mathbf{G}^{\mathrm{T}} \mathbf{G}\right)^{-1} \mathbf{G}^{\mathrm{T}} \mathbf{y}$

where $\mathbf{G}=\left[\begin{array}{llll}1 & G_{1} & \ldots & G_{n}\end{array}\right]$ is the gene response matrix. Since the columns of matrix $\mathbf{G}$ can be collinear, the Moore-Penrose pseudo-inverse $\left(\mathbf{G}^{\mathrm{T}} \mathbf{G}\right)^{\#}$ can be computed using the singular value decomposition instead of the standard matrix inverse $\left(\mathbf{G}^{\mathrm{T}} \mathbf{G}\right)^{-1}$. During the MGGP run, in addition to the standard GP subtree crossover (a low-level crossover), genes can be acquired or deleted using a tree crossover 
operator called high-level crossover. In the low-level crossover, a gene is chosen at random from each 2 parent individual. Then, the standard sub-tree crossover is applied and the created trees will replace the 3 parent trees in the otherwise unaltered individual in the next generation. The high-level crossover 4 allows the exchange of one or more genes with another selected individual subject to the $G_{\max }$ 5 constraint. If an exchange of genes results in any individual containing more genes than $G_{\max }$, the genes 6 will be randomly selected and deleted until the individual contains $G_{\max }$ genes [16-19].

Gene 1

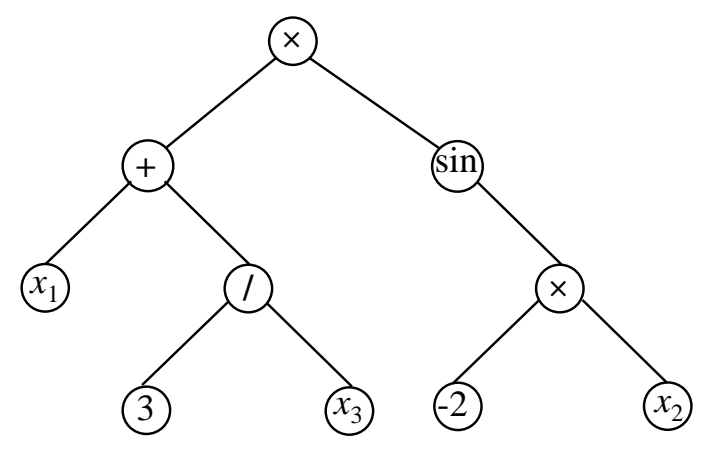

$\left[x_{1}+\left(3 / x_{3}\right)\right] \times \sin \left(-2 \times x_{2}\right)$

\section{Gene 2}

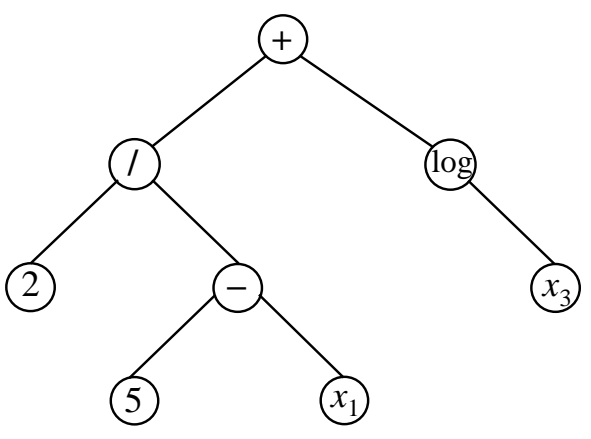

$2 /\left(5-x_{1}\right)+\log \left(x_{3}\right)$

\subsection{Multi-objective genetic programming (MOGP)}

Typically, tree-based standard GP and MGGP optimize a single objective for each individual based on the defined fitness function. In other words, regular tournament selections only consider a fitness function. For symbolic regression, this objective is usually defined as maximizing the goodness-of-fit to the training data. This typically leads to evolution of overly complex, non-robust models due to the tendency (known as "bloat") of acquiring model terms that provide little or no effect on the final prediction. Although more compact models can be developed in MGGP as compared to standard GP [19], MGGP suffers from another type of bloat known as "horizontal bloat" (i.e., the tendency of multigene models to acquire genes that have little or no effect on the fitness). The simplest way to prevent horizontal bloat in multi-gene regression is to limit $G_{\max }$ in a model. In practice, however, it is not easy to judge the optimal value of $G_{\max }$ for any given problem [19]. To deal with this issue, a multiobjective strategy can be implemented into multi-gene symbolic regression, a process known as multiobjective genetic programming (MOGP), which can simultaneously optimize the goodness-of-fit and the complexity of the developed models by searching the so-called Pareto front (non-dominated solutions) set.

Different techniques can be used for conducting multi-objective optimization. In this study, the GPTIPS 2 toolbox [19], in conjunction with subroutines coded in MATLAB, is used to perform MOGP 
through the use of a non-dominated sorting technique [25]. Note that non-dominated sorting technique is used at the end of each generation of the MGGP algorithm to sort the non-dominant solutions based on their complexity and accuracy. First, it classifies the individuals from both the new and old population according to their position on the Pareto front. Pareto front level 1 consists of a set of Pareto optimal solutions that are not dominated by any other solution. The solutions that comprise Pareto front level 2 are not dominated by any other solutions, apart from those in Pareto front level 1, and so on. Next, a "crowding factor" (i.e., the average distance of a solution from the nearest solutions (either side) on the same Pareto front) is calculated for each individual to increase the diversity of the population, giving lower priority to the solutions that are crowded together during the ranking process. Finally, the solutions are ranked according to their position (those on level 1 are ranked above those on level 2, and so on), and the solutions that are on the same Pareto front are ranked according to their crowding factors. The top $50 \%$ of the population will survive to the next generation, while the rest are eliminated [19].

\subsection{Big Data handling in GP}

When either the number of data sets is very large or the data mining is complex enough that traditional methods either cannot handle it or are not practical to employ, we can use the Big Data term. While the number of data sets in engineering studies and especially in experimental studies are generally not very large, they are typically complex [26]. Although GP techniques have been successfully used for engineering system modeling (e.g., [27]), difficulties can arise in dealing with Big Data. In general, statistical data mining approaches such as regression are far faster than evolutionary algorithm approaches such as GP. Even among evolutionary algorithms, GP is one of the slowest techniques because, as previously mentioned, GP also tries to find the structure of the solution(s). Additionally, the multi-objective process is slower than a single-objective process mostly because of the nondominated sorting process. In this study, to accelerate the GP process for Big Data analysis, the following strategies have been used in the GP process:

- Only $60 \%$ of the data have been randomly used for training proposes. To counteract any ill effects of data randomization [28], training sets are randomly chosen for each run;

- The final Pareto front is obtained from merging the Pareto fronts for all runs.

Machine learning algorithms can be classified as either a trajectory-based algorithm (which uses a single solution during the learning process) or a population-based algorithm. Most predictive tools are trajectory-based algorithms such as ANNs and regression analysis. GP is one of the population-based algorithms, where in each generation it deals with a set of solutions (programs). This makes GP flexible enough to adopt with parallel processing, as the MOGP procedure can be accelerated by parallelizing the calculations using a distributed computing machine in order to deal with the Big Data issue in GP. While only twelve cores are used in this study to evolve and evaluate new models, the number of cores can be increased up to the population size using this framework. The schematic of parallel processing in the GP process is shown in Fig. 2. 


\section{3. Applying MOGP to predict the time-dependent total creep for concrete}

5 Concrete creep is defined as the time-dependent increase of strain in hardened concrete (in excess of

Fig. 2. Schematic view of parallel genetic programming. shrinkage) subjected to a sustained stress; it has a direct influence on prestress losses of pretensioned concrete members and the long-term deflection of girders [29]. Furthermore, it is well known that in concrete repairs, cracking due to restrained shrinkage can reduce the performance of a structure and create a direct path for penetration of corrosive ions into the concrete that can in turn reduce the service life performance of the structure. Creep can significantly reduce the shrinkage-induced tensile stresses and delay or prevent the formation of cracks in repaired concrete [30-36].

For concrete, total creep includes an instantaneous component, basic creep, and drying creep. When a load is applied on a concrete specimen, the specimen first shows a recoverable instantaneous deformation. Basic creep occurs in specimens that are sealed to prevent the ingress or egress of moisture. Drying creep is the additional creep caused by drying. To describe concrete creep, a creep coefficient or a compliance function is generally used. The creep coefficient $\phi\left(t, t_{0}\right)$ is the ratio of the creep strain to the initial strain, while the compliance $J\left(t, t_{0}\right)$ is the total load-induced strain (elastic strain plus creep strain) at age $t$ per unit stress caused by a unit uniaxial sustained load applied since loading age $t_{0}$. In calculating $\phi\left(t, t_{0}\right)$, the elastic modulus of the concrete should be determined or

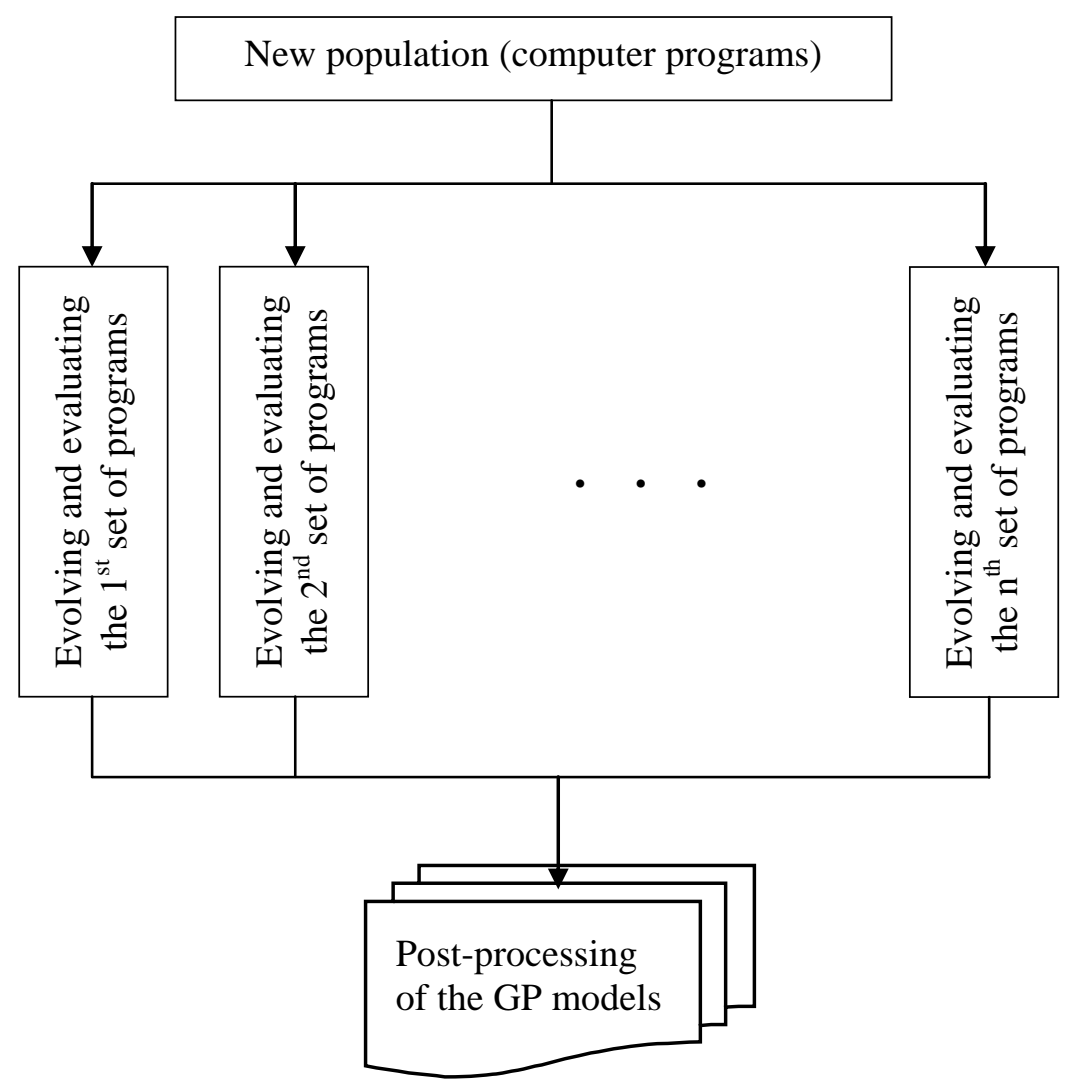


measured. Due to errors in determining or measuring the elastic modulus of concrete, it is recommended to report the creep values based on the compliance function instead of the creep coefficient [37].

In this section, the application of MOGP is illustrated through the development of a practical nonlinear equation for predicting the time-dependent total creep compliance of concrete. First, the model development procedure and the parameter settings used for conducting the MOGP are described. Next, the properties of the experimental data used for model development are presented. The optimum developed MOGP model is then selected for predicting the time-dependent concrete total creep compliance. Lastly, the accuracy of the developed model is compared with previously developed wellknown creep prediction models.

\subsection{Model development using MOGP}

Different parameters affect the total creep of concrete. In this study, the input variables (x) used in the proposed MOGP models are selected based on the parameters in the Bažant-Baweja B3 model [38] for predicting the time-dependent total creep compliance of concrete - excluding the cement type, curing method and period, elastic modulus of concrete (due to its correlation with concrete compressive strength), and section shape [38]. Consequently, nine variables are selected as input variables (x): water-to-cement ratio $(w / c)\left(\mathrm{kg} / \mathrm{m}^{3}\right)$, water content, $(w)\left(\mathrm{kg} / \mathrm{m}^{3}\right)$, cement content $(c)\left(\mathrm{kg} / \mathrm{m}^{3}\right)$, aggregate to cement ratio by weight $(\mathrm{a} / \mathrm{c})$, concrete mean compressive strength at 28 days $\left(f_{\mathrm{c}}^{\prime}\right)(\mathrm{MPa})$, volume-tosurface ratio $(V / S)(\mathrm{mm})$, age of concrete at loading $\left(t_{0}\right)$ (day), ambient relative humidity $(h)(\%)$, and time since the application of load $\left(t_{e}\right)$ (day). These input variables should be used in MOGP for selecting the most contributed variables and developing the prediction models. It should be noted that the effect of the cement type on $J\left(t, t_{0}\right)$ will be considered later in the model by re-evaluating the coefficients $\left(d_{i}\right)$ of the final MOGP model.

To increase the model development capability of MOGP, the appropriate parameters should be used in the MOGP predictive algorithm. Basic arithmetic operators and mathematical functions are adopted to obtain the optimum MOGP models. The models are formed by randomly combining the elements from the functional set and the terminal set. The number of programs in the population is determined by the population size, and the number of generations determines the number of levels the algorithm will use before the run terminates. The values for the population size and the number of generations are set based on the nature of the data set, the number of variables to be considered, and the complexity of problem. Note that the size and various forms of the model to be searched for in the global solution space are determined by the maximum number of genes allowed in an individual $\left(G_{\max }\right)$ and the maximum tree depth $\left(D_{\max }\right)$. For posing a trade-off between the running time and the complexity of the evolved solutions, $G_{\max }$ and $D_{\max }$ are set to the optimal values of 3 and 5 , respectively. Table 1 shows the parameter settings used for the MOGP implementation in this study. The parameter settings used in Table 1 are based on previously suggested values that can be found in the literature [16-19], and employing a trial-and-error approach. 
Table 1. Parameter settings for MOGP algorithm

\begin{tabular}{cc}
\hline Parameter & Settings \\
\hline Function set &,,$+- \times, /, \sqrt{ }$, exp, ln, sin, cos, tanh \\
Population size & $100-500$ \\
Number of generations & $500-1000$ \\
Maximum number of genes allowed in an individual $\left(G_{\max }\right)$ & 3 \\
Maximum tree depth $\left(D_{\max }\right)$ & 5 \\
Tournament size & $10 \%$ of population \\
Crossover events & 0.85 \\
High-level crossover & 0.2 \\
Low-level crossover & 0.8 \\
Mutation events & 0.12 \\
Sub-tree mutation & 0.9 \\
Replacing input terminal with another random terminal & 0.05 \\
Gaussian perturbation of randomly selected constant & 0.05 \\
Direct reproduction & 0.05 \\
Ephemeral random constants & {$[-1010]$} \\
\hline
\end{tabular}

Here, a "rate-based high-level crossover" through the use of a crossover rate parameter $(C R)$ is employed to generate new genes for individuals as well as to reduce the overall number of genes for one model and increase the total number of genes for the other. A uniform random number $0<r<1$ with a default value of 0.5 is generated independently for each gene in the parents. If $r<C R$, then the corresponding gene is moved to the other individual. If an exchange of genes leads to offspring that contain more genes than the $G_{\max }$, then the genes are randomly deleted until the constraint is no longer violated [19].

For the analyses, the data are randomly split into a training subset and a validation subset. The training subset consists of a set of data used only for learning (to fit the genetic model evolution). The validation subset is used to measure the performance of the programs evolved by MOGP on data that played no role in building the models. In order to obtain a consistent data division, several combinations of training and testing sets are considered. As mentioned earlier, the fitness function evaluates the evolved expressions to designate the best encoded expressions. For the MGGP fitness function, the minimum root mean square error (RMSE) between the measured and predicted output is defined by:

$R M S E=\sqrt{\frac{\sum_{i=1}^{i=n}\left|y_{i}-\hat{y}_{i}\right|^{2}}{n}}$

where $y_{i}$ and $\hat{y}_{i}$ are the measured and calculated output values for the $i^{\text {th }}$ output, respectively, and $n$ is the number of samples. The best models are selected based on a MOGP approach through simultaneous optimization of two objectives: maximizing the correlation coefficient $(R)$ and minimizing the model complexity.

\subsection{Experimental Data}


1 Comprehensive experimental data selected from the NU-ITI data bank is used for development of the 2 time-dependent creep model [22]. This data bank contains 1403 creep specimens and 1809 shrinkage 3 specimens. The data selected for the model development include only the total creep specimens of 4 concrete without pozzolans that are exposed to mean ambient temperatures from $20^{\circ} \mathrm{C}$ to $25^{\circ} \mathrm{C}$ and are 5 subjected to a sustained stress that is less than $50 \%$ of the mean concrete strength at the time of loading 6 (i.e., service stress range); any drying, underwater, and basic creep specimens are excluded. For 7 selected specimens, data for certain variables was missing or incomplete. Any specimens having more 8 than two variables out of seven $\left(w / c, w\right.$ or $c, a / c, f_{\mathrm{c}}^{\prime}, V / S, t_{0}$, and $\left.h\right)$ that were not reported in the data 9 bank were excluded. For specimens with one or two missing variables, the multiple imputation method $10[23,39]$ is adopted in this paper to handle the missing data. This approach gives a consistent, asymptotically efficient and normal estimation, and it allows missing data to be handled in a way that is unbiased and statistically valid [40, 41]. The properties of the final selected specimens after addressing the missing data issue are summarized in Table 2. References used for collecting Big Data in Table 2 can be found in Electronic Annex in the online version of this paper. Note that the experimental result for the concrete creep compliance of each specimen is a time-series, which records the compliance at different time moments. The number of data points listed in Table 2 refers to the number of times the compliance is recorded.

Table 2. Properties of the selected specimens

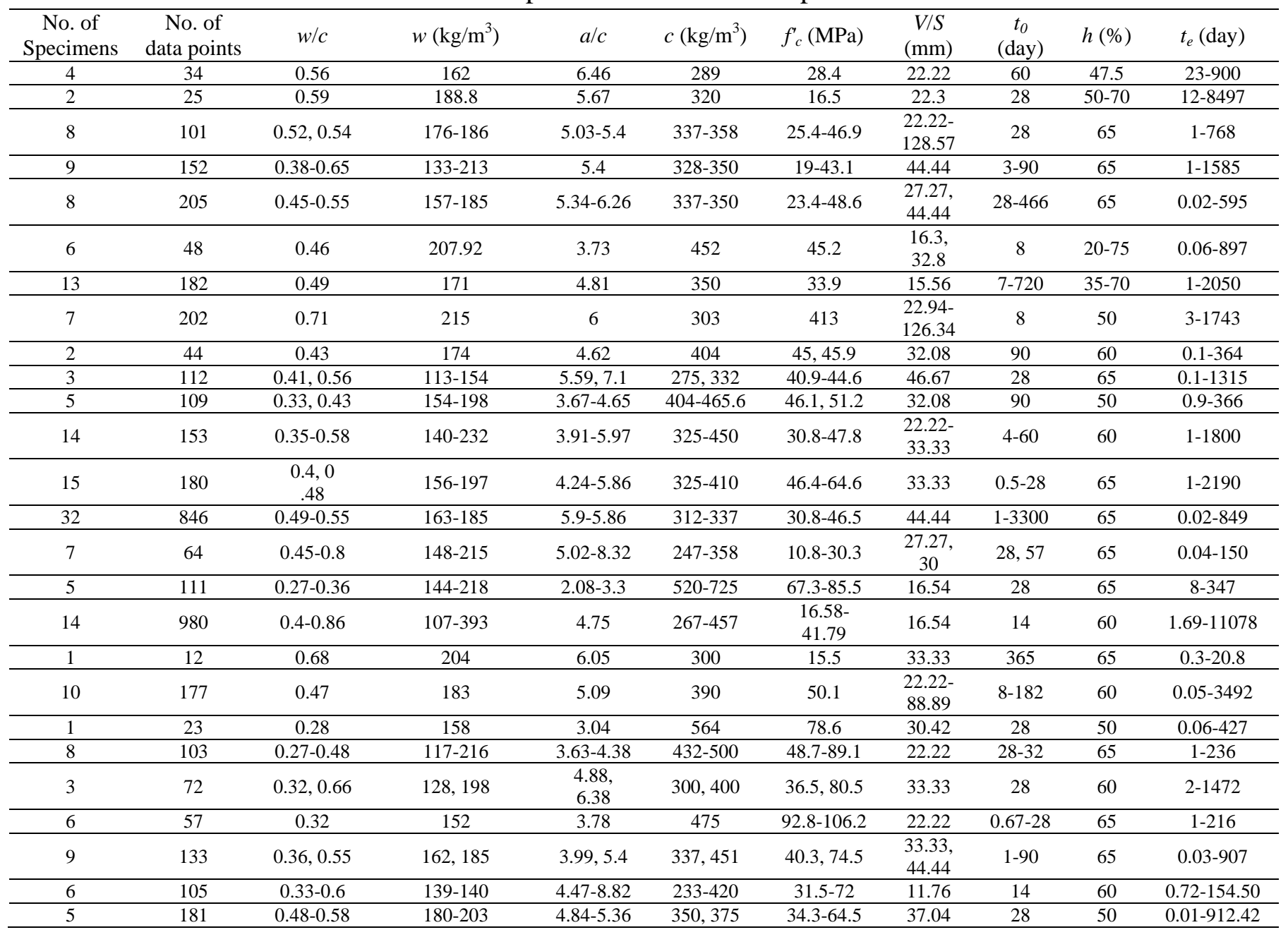




\begin{tabular}{|c|c|c|c|c|c|c|c|c|c|c|}
\hline 1 & 33 & 0.44 & 180 & 3.75 & 410 & 49.2 & 37.04 & 28 & 65 & $0.01-238.88$ \\
\hline 4 & 184 & $0.47-0.57$ & $169-200$ & $4.6-5.13$ & 350,360 & $41-53$ & 27.27 & 7 & 50 & $0.01-90.06$ \\
\hline 4 & 44 & $0.32-0.39$ & $140-159$ & $4.03-5.4$ & $361-457$ & $53.4-71.2$ & 30 & 7 & 70 & $0.2-48.2$ \\
\hline 4 & 112 & 0.33 & 156 & 3.75 & 473 & 48.7 & 21.43 & $3-90$ & 50 & $0.3-195$ \\
\hline 4 & 58 & $0.33,0.38$ & 150,163 & $\begin{array}{l}1.75 \\
1.86 \\
\end{array}$ & 430,455 & $50-95$ & 12.6 & 2,28 & 55 & $1-1375$ \\
\hline 2 & 45 & 0.4 & 137 & 5.6 & 343 & 27.9 & 33.33 & 28,96 & 50 & $0.2-570$ \\
\hline 2 & 59 & 0.4 & 137 & 5.6 & 343 & 44.5 & 33.33 & 28,96 & 50 & $0.1-568.2$ \\
\hline 3 & 60 & $0.35,0.4$ & 137,168 & $\begin{array}{l}3.57 \\
5.59\end{array}$ & 343,480 & 43.61 & 33.33 & $28-96$ & 50,57 & $0.22-1199.2$ \\
\hline 2 & 21 & 0.43 & 157 & 4.96 & 365 & 50.5 & 31.58 & 923 & 20 & $1.13-405.66$ \\
\hline 1 & 12 & 0.53 & 177 & 5.49 & 334 & 46.75 & 12.5 & 30 & 50 & $0.01-111.65$ \\
\hline 5 & 49 & $0.49,0.52$ & 161,171 & $\begin{array}{c}5.63, \\
5.71\end{array}$ & 329 & $\begin{array}{l}41.57, \\
44.49\end{array}$ & $\begin{array}{c}30, \\
33.33\end{array}$ & 90 & 45,57 & $0.27-393.6$ \\
\hline 2 & 69 & 0.38 & 175 & 3.74 & 460 & 39.79 & 33.33 & 63 & 57 & $0.01-155.59$ \\
\hline 2 & 26 & 0.4 & 160 & 4.45 & 400 & 55.47 & 22.5 & 30,100 & 65,67 & $0.24-269.23$ \\
\hline 29 & 200 & $0.24-0.57$ & $168-171$ & $0.79-6.05$ & $300-700$ & $\begin{array}{l}38.29- \\
112.27\end{array}$ & 32.77 & 3,28 & 50,61 & $1-1250$ \\
\hline 1 & 34 & 0.51 & 189 & 4.37 & 370 & 33.71 & 33.33 & 7 & 60 & $0.05-162.2$ \\
\hline 1 & 12 & 0.44 & 162.8 & 4.37 & 370 & 39.69 & $\begin{array}{l}27.88- \\
28.79 \\
\end{array}$ & 7 & 60 & $0.25-181.70$ \\
\hline 1 & 8 & 0.53 & 176 & 5.29 & 332 & 39.67 & 18.94 & 28 & 63 & $0.04-0.33$ \\
\hline 7 & 258 & $0.43,0.5$ & $159-203$ & $4.07-5.11$ & $360-406$ & $\begin{array}{c}30.76- \\
50.67 \\
\end{array}$ & $\begin{array}{c}22.73- \\
33 \\
\end{array}$ & 14,91 & 60,82 & $1-522.4$ \\
\hline 6 & 6 & 0.38 & 167 & 4.03 & 440 & 89.83 & 22.22 & 28 & 60 & 730 \\
\hline 1 & 32 & 0.6 & 169 & 6.38 & 282 & 25.79 & 22.22 & 7 & 60 & $0.1-93$ \\
\hline 1 & 16 & 0.4 & 142 & 5.28 & 355 & 45.4 & 20 & 28 & 60 & $0.02-4000$ \\
\hline 11 & 74 & $0.3-0.62$ & $170-173$ & $2.89-6.89$ & $274-567$ & $30.3-79$ & 20 & 28 & 60 & $1-730$ \\
\hline 3 & 45 & 0.35 & 150,155 & $1.49-2.69$ & 430,443 & $32.7-55.4$ & 20 & 17 & 60 & $2-303$ \\
\hline 5 & 5 & $0.2-0.3$ & $133-140$ & $2.48-4.17$ & $444-665$ & $78.1-117$ & 20 & 1 & 60 & 7 \\
\hline 8 & 87 & 0.3 & 151 & $\begin{array}{l}3.49 \\
3.54\end{array}$ & 505 & $\begin{array}{l}68.43, \\
91.04\end{array}$ & 30 & $7-28$ & 57.5 & $3-284$ \\
\hline 3 & 26 & $0.37,0.4$ & 144,156 & $1.67-2.18$ & 390 & $35-40.3$ & 20 & 28 & 60 & $7-1090$ \\
\hline 2 & 20 & $0.46,0.57$ & 141,160 & $\begin{array}{c}6.16, \\
6.65\end{array}$ & 280,306 & $\begin{array}{l}36.63, \\
45.45\end{array}$ & 22.22 & 28 & 50 & $7-434$ \\
\hline 12 & 30 & $0.43,0.48$ & 182,209 & $\begin{array}{l}3.12, \\
4.46\end{array}$ & 379,486 & $\begin{array}{l}43.86, \\
48.11 \\
\end{array}$ & 22.22 & 7,28 & $40-70$ & $0.13-98$ \\
\hline 2 & 21 & $0.3,0.5$ & 160,170 & $\begin{array}{l}3.24, \\
5.31\end{array}$ & 340,533 & $71,32.2$ & 22.22 & 28 & 60 & $2-360$ \\
\hline 3 & 51 & $0.38-0.9$ & $158-193$ & $4.51-9$ & $214-415.2$ & $15.2-31.9$ & $\begin{array}{l}5.64- \\
10.69\end{array}$ & $10-60$ & 50 & $0.03-180$ \\
\hline 1 & 6 & 0.39 & 175.5 & 4.05 & 460 & 71.2 & 22.73 & 3 & 68 & $1.93-30.03$ \\
\hline 2 & 18 & 0.3 & 171 & 2.99 & 570 & 65.4 & 30 & 28 & 50 & $1.01-581.42$ \\
\hline 4 & 100 & $0.28,0.33$ & $124-126$ & 4-4.71 & $383-450$ & $73.95-88.4$ & 24.21 & 28 & 65 & $0.57-201.92$ \\
\hline 12 & 96 & $0.43-0.67$ & $163-201$ & $1.22-4.02$ & $270-380$ & 19.71-41.1 & 30 & 28 & 50 & $1-3000$ \\
\hline 1 & 11 & 0.26 & 156 & 2.91 & 600 & 81 & 21.43 & 28 & 57 & $0.87-180.81$ \\
\hline 11 & 371 & $0.32-0.38$ & $145-172$ & $\begin{array}{l}3.77, \\
3.96\end{array}$ & 450,453 & $69.5-87.33$ & 34.48 & $0.6-28$ & 50,99 & $0.01-202.82$ \\
\hline 15 & 566 & $0.41-0.69$ & $159-225$ & $2.95-7.15$ & 275,550 & $40.5-64.6$ & 14.89 & 3 & 65,99 & $0.15-91.95$ \\
\hline 4 & 70 & $0.29,0.52$ & 145,182 & $3.6,5.25$ & 350,500 & $59.1-87$ & 20.6 & 7 & 45,60 & $0.2-367.68$ \\
\hline 6 & 73 & $0.32-0.87$ & $178-193$ & $2.81-8.6$ & $220-557$ & $21.6-59.8$ & 20.38 & 28 & 61 & $0.06-60.61$ \\
\hline 1 & 15 & 0.28 & 183.4 & 2.41 & 655 & 71.4 & 30.42 & 28 & 57 & $0.8-362.7$ \\
\hline 1 & 4 & 0.28 & 183.4 & 2.41 & 655 & 71.4 & 30.42 & 28 & 57 & $23.16-156.5$ \\
\hline
\end{tabular}

1

2 Overall, a total number of 393 specimens and 7438 corresponding data points were selected, 3 which presents a Big Data challenge for analyzing concrete total creep compliance. The variable ranges 4 of the selected specimens used for the model development based on Table 2 are: $0.2 \leq w / c \leq 0.9,106$ $5 \mathrm{~kg} / \mathrm{m}^{3} \leq w \leq 393 \mathrm{~kg} / \mathrm{m}^{3}, 0.8 \leq a / c \leq 9,214 \mathrm{~kg} / \mathrm{m}^{3} \leq c \leq 725 \mathrm{~kg} / \mathrm{m}^{3}, 11 \mathrm{MPa} \leq f^{\prime}{ }_{c} \leq 117 \mathrm{MPa}, 5.6 \leq V / S \leq$ 6 128.6, 0.5 day $\leq t_{0} \leq 3300$ day, $20 \% \leq h \leq 99 \%$, and 0.01 day $\leq t_{e} \leq 11078$ day). The data for the 7 selected specimens shows a wide range of properties; consequently, the proposed model based on this 8 database will be valid for a wide range of structural properties and can be used for predicting the total 9 concrete creep compliance in both the short term and the long term (up to 11078 days). According to 
ACI 363R-92 [42], concrete with $f_{c}^{\prime}>41 \mathrm{MPa}$ can be classified as high-strength concrete (HSC). Based on the properties of selected specimens in Table 2, it can be ascertained that $57 \%$ of the selected specimens are HSC specimens and 43\% are normal strength concrete (NSC) specimens. Therefore, the proposed G-C model can also be used for total creep prediction of both NSCs and HSCs, while most models in the literature are valid only for creep prediction of NSCs [30] and have limitations in their application (e.g., ACI 209R-92 model 1992 is only developed for type I and type III cements [37]).

\subsection{Results and discussion}

As mentioned in section 4.1, cement type is one of the important parameters that can affect the total creep of concrete. For each cement type, one could develop a separate prediction model based on the experimental data for specimens of that cement type. While it is quite possible that the obtained model formulations would be different for different cement types, it is more practical to adopt the same formulation structure for all cement types. Therefore, in this study, the entire database (which contains three cement types: CEB Type R cement, CEB Type SL cement, and CEB Type RS cement) is used to develop a single prediction model for concrete creep. The final model formulation is selected based on a compromise between the prediction accuracy (as measured by the coefficient of determination $R^{2}$ ) and the model complexity (as measured by the number of input variables). Next, the entire database is divided into three groups based on the three types of cement. Using each group of data, the predicted coefficients of the final model $\left(d_{i}\right)$ are re-evaluated by conducting the regression analysis to reflect the influence of the cement type. Through MOGP, the final formulation of the proposed G-C model for the prediction of time-dependent total creep compliance of concrete is obtained as follows:

$\hat{J}\left(t, t_{0}\right)=d_{0}+d_{1} G_{1}+d_{2} G_{2}+d_{3} G_{3}$

$G_{1}=\left(\frac{w}{c}\right) \cdot \frac{\ln \left(t_{e}+2.46\right)}{f_{c}^{\prime}}$

$G_{2}=\ln \left[\left(\frac{w}{c}\right) \sqrt{t_{0}}\right]$

$G_{3}=\left(\frac{f_{c}^{\prime} \cdot t_{0}}{h^{2}}\right)^{2}$

As shown in Eqs. (4) to (7), the proposed G-C model has a rather simple and compact formulation when compared with other well-known creep prediction models (such as the ACI 209R-92 model [43] and the Bažant-Baweja B3 model [38]), and it incorporates only five out of the nine input variables used in the model development process. The contribution of each predictor variable in the developed concrete creep prediction models can be evaluated through their frequencies, in which a frequency value of 1.0 for a variable indicates that it has been selected in $100 \%$ of the best generated models [44]. Note that in this study, we assume the models with $R^{2} \geq 0.6$ are the best generated models. The 
1 frequency histograms of the input variables are shown in Fig. 3. As can be seen in this figure, for the 2 collected database in this study, $f_{c}^{\prime}$ and $V / S$ have the most and the least statistically significant 3 contributions in the best generated MOGP models, respectively. Consequently, $V / S$ was not selected as 4 an input variable in the model development process of the G-C model.

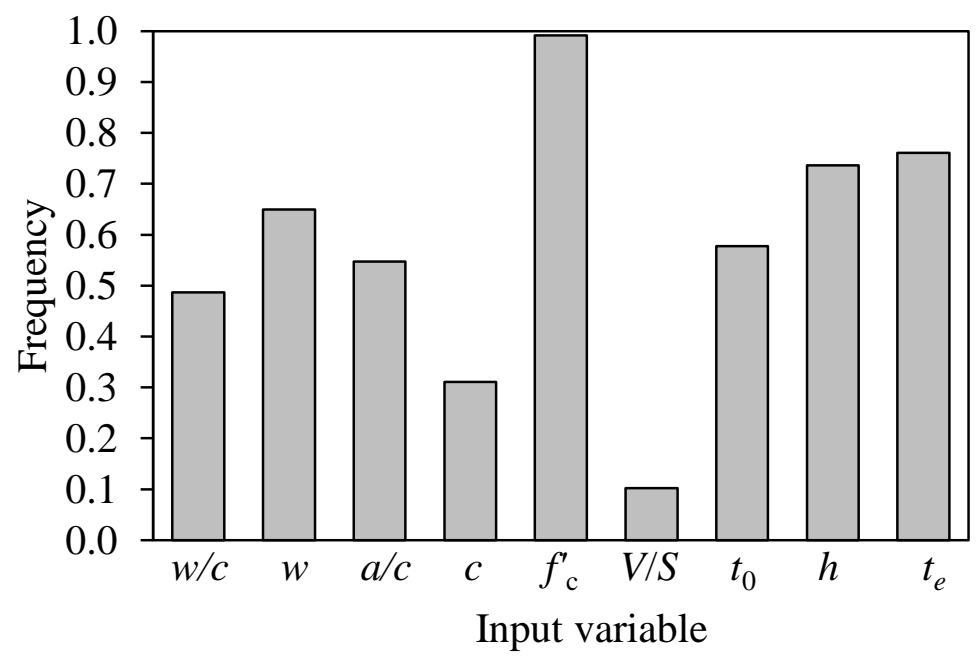

Fig. 3. Histograms of the frequency of each variable used in the best generated MOGP models

The results of all models developed by MGGP (shown in solid blue circles), the optimized models in the Pareto front selected using a non-dominated sorting method (shown in solid green circles), and the adopted G-C model (encircled in red) are presented in Fig. 4. As mentioned earlier, the Pareto front is obtained by using a non-dominated sorting method at the end of a MOGP run through simultaneously optimizing the accuracy and the complexity of the developed models.

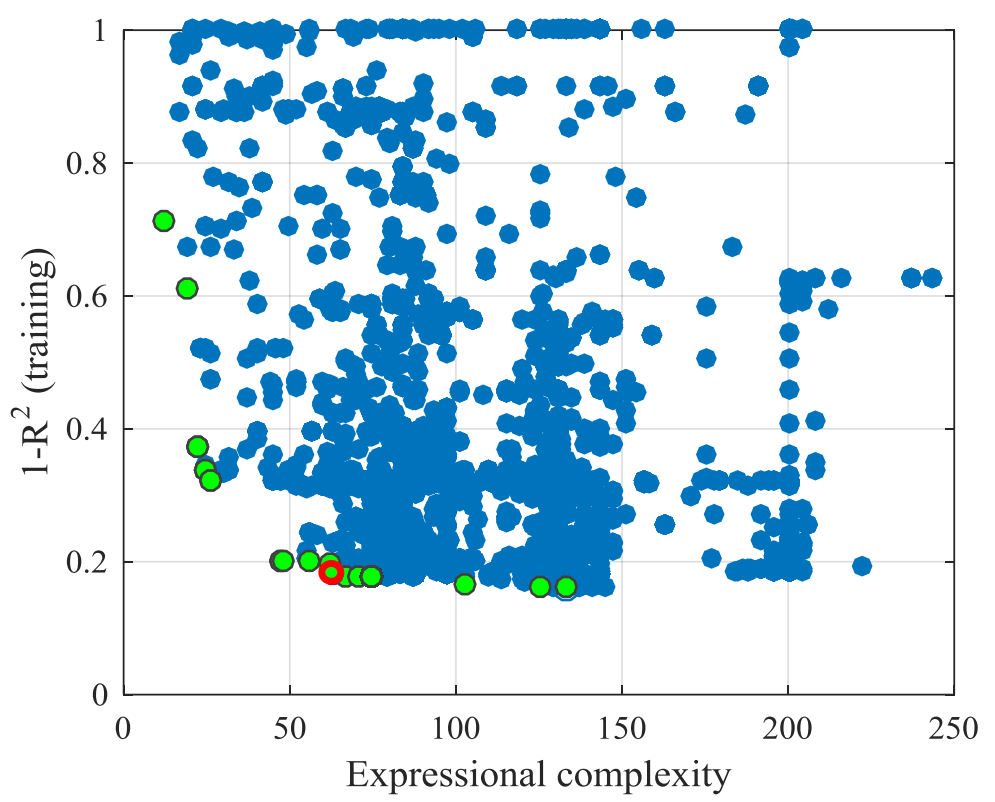

Fig. 4. All models developed by MGGP (solid blue circles), Pareto front results obtained by nondominated sorting (solid green circles), and the selected G-C model (encircled in red) 
With the formulation of Eq. (4), the model parameters $d_{i}$ are re-evaluated based on the data for the three specific types of cement. Table 3 shows the results obtained for the final model parameters when considering the effect of cement type.

Table 3. Evaluation results for model parameters using Eq. (4)

\begin{tabular}{|c|c|c|c|c|c|c|c|}
\hline \multirow{2}{*}{$\begin{array}{c}\text { Cement } \\
\text { type }\end{array}$} & \multirow{2}{*}{ Parameter } & \multirow{2}{*}{ Mean } & \multirow{2}{*}{ Standard error } & \multicolumn{4}{|c|}{ Correlation Coefficient } \\
\hline & & & & $d_{0}$ & $d_{1}$ & $d_{2}$ & $d_{3}$ \\
\hline \multirow{4}{*}{$\mathrm{R}$} & $d_{0}$ & 26.43 & 0.71 & 1 & & & \\
\hline & $d_{1}$ & 1038.37 & 6.91 & -0.39 & 1 & & \\
\hline & $d_{2}$ & -10.27 & 0.80 & -0.46 & -0.35 & 1 & \\
\hline & $d_{3}$ & 0.0235 & 0.01 & 0.21 & 0.25 & -0.67 & 1 \\
\hline \multirow{4}{*}{$\mathrm{RS}$} & $d_{0}$ & 30.16 & 1.02 & 1 & & & \\
\hline & $d_{1}$ & 936.90 & 17.75 & -0.67 & 1 & & \\
\hline & $d_{2}$ & -17.59 & 1.10 & -0.26 & -0.30 & 1 & \\
\hline & $d_{3}$ & 0.0022 & 0.004 & 0.1 & 0.23 & -0.71 & 1 \\
\hline \multirow{4}{*}{ SL } & $d_{0}$ & 53.55 & 2.36 & 1 & & & \\
\hline & $d_{1}$ & 695.20 & 25.13 & -0.65 & 1 & & \\
\hline & $d_{2}$ & -20.66 & 1.32 & -0.56 & -0.08 & 1 & \\
\hline & $d_{3}$ & 0.04 & 0.001 & 0.05 & 11 & -0.32 & 1 \\
\hline
\end{tabular}

A number of prediction models for concrete total creep compliance have been previously published in various reports and building code designs. To further evaluate the prediction performance of the proposed G-C model, it seems worthwhile to compare the prediction results obtained from the previously developed models with those from the proposed G-C model. Some of the well-known models in this regard are the ACI 209R-92 model [43], the Bažant-Baweja B3 model [38], the CEB MC90-99 model [45], and the GL2000 model [46]. However, the ACI 209R-92 model [43] is only valid for type I and type III cements and it requires additional parameters such as concrete slump, the ratio of fine aggregate to total aggregate by weight, and air content, which are not reported in the NU-ITI data bank. In addition, this model proposes a creep coefficient instead of creep compliance, which may introduce problems due to the assumed value of the elastic modulus [37]. Therefore, the ACI 209R-92 model is not considered for the comparison in this study.

The complexity and accuracy of the models are assessed and compared using different quantitative measures and by plotting the predictions and the measured data. In this study, we use a number of input variables to compare the complexity along with $R^{2}, R R M S E, \rho$ [47], and three other statistical indicators proposed by CEB MC90-99 (as found in Appendix B of [37]) - CEB coefficient of variation $V_{C E B}$, CEB mean square error $F_{C E B}$, and CEB mean deviation $M_{C E B}$ - to evaluate the accuracy of the prediction models. Table 4 shows the $R^{2}, R R M S E, V_{C E B}, F_{C E B}$, and $M_{C E B}$ values obtained for each model.

Table 4. Comparison of complexity and accuracy of the prediction models

\begin{tabular}{cccccccc}
\hline \multirow{2}{*}{ Model } & Number of input & \multicolumn{5}{c}{ Accuracy measure } \\
\cline { 3 - 7 } & variables* & $R^{2}$ & $R R M S E$ & $\rho$ & $V_{C E B}$ & $F_{C E B}$ & $M_{C E B}$ \\
\hline G-C & $\mathbf{5}^{* * *}$ & $\mathbf{0 . 8 3}$ & $\mathbf{0 . 3 7}$ & $\mathbf{0 . 1 9}$ & $\mathbf{0 . 3 7}$ & 61.7 & $\mathbf{1 . 6 3}$ \\
Bažant-Baweja B3 [38] & 10 & 0.56 & 0.62 & 0.35 & 0.59 & $\mathbf{5 5 . 7}$ & 2.10 \\
CEB MC90-99 [45] & $\mathbf{5}$ & 0.62 & 0.55 & 0.31 & 0.54 & 67.4 & 1.73 \\
GL2000 [46] & 6 & 0.48 & 0.69 & 0.41 & 0.65 & 57.9 & 2.22 \\
\hline
\end{tabular}

* The type of cement, curing method and cross-section shape are not considered as input variables. 
** The best results in each column are indicated in bold type.

As shown in Table 4, the proposed G-C model uses only five variables for predicting concrete creep, indicating that the G-C model is simple. In addition, the G-C model gives the highest value of $R^{2}$ and the generally lowest values of RRMSE, $\rho, V_{C E B}$, and $M_{C E B}$, indicating that the G-C model is more accurate than the CEB MC90-99 [45], Bažant-Baweja B3 [38], and GL2000 [46] models. Fig. 5 shows the predictions for total creep compliance of concrete obtained from the proposed G-C model, from the other three models, and from the experimental data. For an ideal prediction, all data points will fall on the 45-degree line (i.e., the equality line, which is indicated as a solid grey line in Fig. 5). The dashed red lines show the $95 \%$ prediction intervals (upper and lower bounds), corresponding to \pm 1.96 standard deviations of the prediction error for the proposed prediction model. It can be seen that the data points obtained from the proposed G-C model are evenly scattered around the solid grey line, with most points inside the dashed red lines, indicating that the proposed G-C model provides unbiased predictions with sufficient accuracy.

Compared with the G-C model, both the Bažant-Baweja B3 [38] and the GL2000 [46] models have the tendency to provide underestimated predictions for high creep values, and the GL2000 model [46] has the tendency to provide overestimated predictions for low creep values. In addition, the scatter in the plot of the CEB MC90-99 model [45] is also evenly spread along the equality line, but it is larger than the scatter in the plot of the G-C model, indicating that the CEB MC90-99 model [45] predictions are less accurate than those of the G-C model. Note that the prediction intervals are not shown in Fig. 5 for the other models, since the standard deviations of the prediction errors are not reported. Therefore, it can be concluded from the results that the proposed MOGP technique can be used as a suitable tool for developing compact and accurate models in the case of nonlinear complex civil engineering systems. 

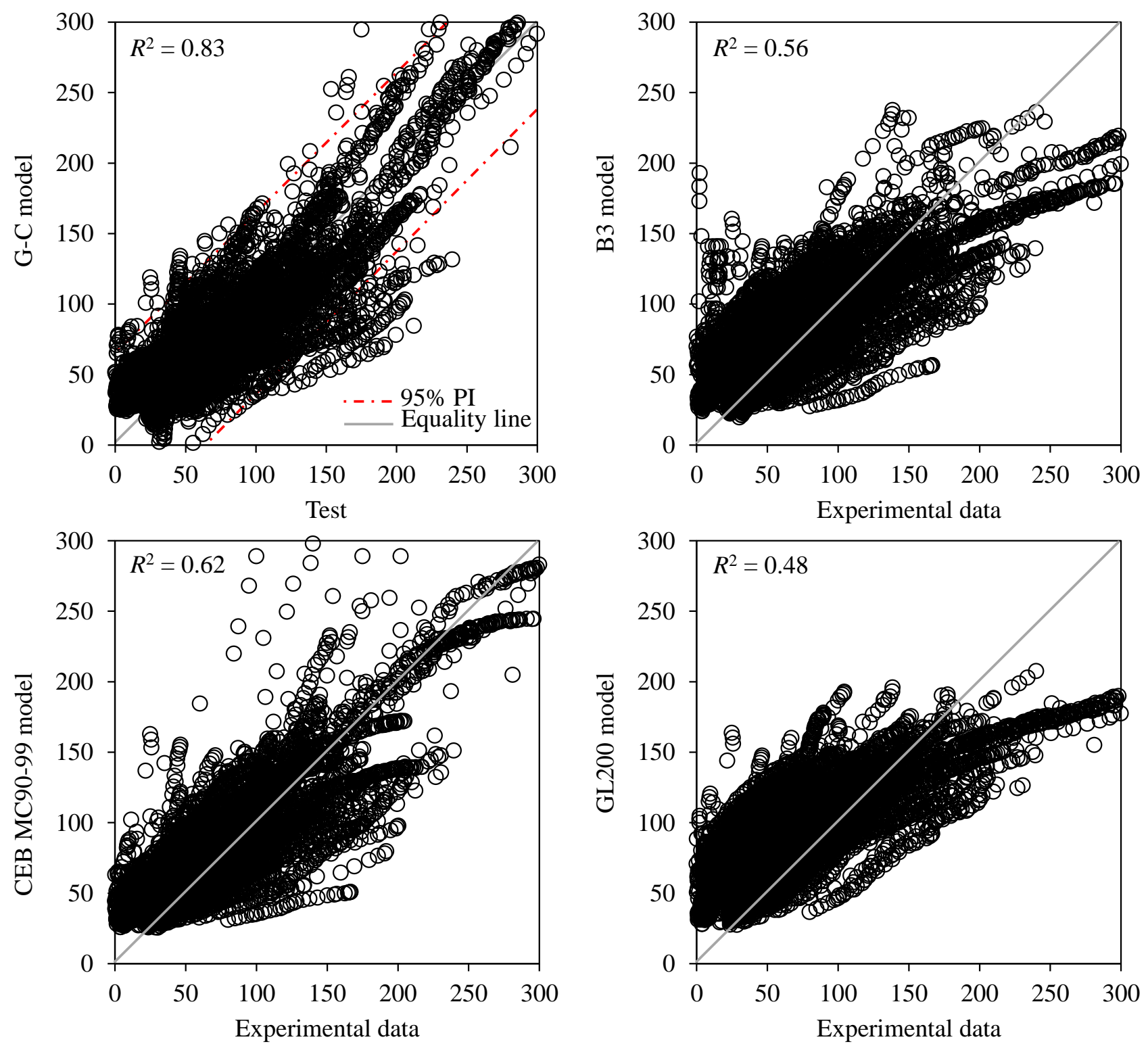

Fig. 5. Prediction of the total creep compliance of concrete, $J\left(t, t_{0}\right)\left(10^{-6} / \mathrm{MPa}\right)$, obtained from the G-C, Bažant-Baweja B3 [38], CEB MC90-99 [45], and GL2000 [46] models versus experimental data

\section{Summary and Conclusions}

This paper proposes a multi-objective genetic programming (MOGP) technique for the modeling of complex engineering systems. The proposed technique can automatically select the most significant variables in the model, formulate the model structure, and solve the unknown parameters of the regression equation, while simultaneously optimizing for both accuracy and complexity. To handle Big Data, some schemes (such as parallel processing) have been used in the proposed MOGP technique. As an illustration, this technique is applied to develop a prediction model for the time-dependent total creep compliance of concrete, which is a complex engineering system. A Big Data selected from the NU-ITI 
database is used for the model development. After conducting MOGP, the optimum genetic programming based creep model (referred to as the "G-C model" in this paper) is selected from among the generated candidate models based on a trade-off between the accuracy and the model complexity. Based on the results, the following conclusions are drawn:

1. For the selected database, the results show that concrete compressive strength and volume-tosurface ratio have the most and the least statistically significant contributions in the best generated MOGP models, respectively.

2. The MOGP generated G-C model for predicting total creep compliance of concrete has a simple, compact formulation including the important parameters that have been identified in the literature (e.g., the water-to-cement ratio and the type of cement).

3. The comparison between the total creep compliance values predicted by the G-C model and the experimental results collected from the literature shows that the proposed creep model provides sufficiently accurate (with $R^{2}=0.83$ ) and unbiased predictions.

4. When compared to the Bažant-Baweja B3 [38], CEB MC90-99 [45], and GL2000 [46] models using various statistical indicators, the $\mathrm{G}-\mathrm{C}$ model provides more accurate predictions for the selected database.

5. For the database used in this study, both the Bažant-Baweja B3 [38] and GL2000 [46] models underestimate the predictions for high creep values, while the GL2000 [46] model overestimates the predictions for low creep values.

6. The MOGP technique proposed in this paper is shown to be a suitable tool for handling Big Data, and it can be used to generate compact and accurate models for complex nonlinear civil engineering systems.

\section{Acknowledgments}

This material is based in part upon work supported by the National Science Foundation (NSF) under Cooperative Agreement No. DBI-0939454. Any options, findings, and conclusions or recommendations expressed in this material are those of the authors and do not necessarily reflect the views of NSF.

\section{References}

[1] Walter E, Pronzato L. Identification of parametric models. Communications and Control Engineering. 1997;8.

[2] Metenidis MF, Witczak M, Korbicz J. A novel genetic programming approach to nonlinear system modelling: application to the DAMADICS benchmark problem. Engineering Applications of Artificial Intelligence. 2004;17:363-70.

[3] Gandomi AH, Alavi AH. A new multi-gene genetic programming approach to nonlinear system modeling. Part I: materials and structural engineering problems. Neural Computing and Applications. 2012;21:171-87.

[4] Gandomi AH, Alavi AH. A new multi-gene genetic programming approach to non-linear system modeling. Part II: geotechnical and earthquake engineering problems. Neural computing and applications. 2012;21:189-201. 
[5] Karthikeyan J, Upadhyay A, Bhandari NM. Artificial neural network for predicting creep and shrinkage of high performance concrete. Journal of Advanced Concrete Technology. 2008;6:135-42.

[6] Motamedi S, Shamshirband S, Hashim R, Petković D, Roy C. Estimating unconfined compressive strength of cockle shell-cement-sand mixtures using soft computing methodologies. Engineering Structures. 2015;98:49-58.

[7] Moini M, Lakizadeh A, Mohaqeqi M. Effect of mixture temperature on slump flow prediction of conventional concretes using artificial neural networks. Australian Journal of Civil Engineering. 2012;10:87-98.

[8] Moini M, Lakizadeh A. Concrete Workability: An Investigation on Temperature effects Using Artificial Neural Networks: AuthorHouse; 2011.

[9] Agatonovic-Kustrin S, Beresford R. Basic concepts of artificial neural network (ANN) modeling and its application in pharmaceutical research. Journal of pharmaceutical and biomedical analysis. 2000;22:717-27.

[10] Gandomi AH, Roke DA. Assessment of artificial neural network and genetic programming as predictive tools. Advances in Engineering Software. 2015;88:63-72.

[11] Karamizadeh S, Abdullah SM, Halimi M, Shayan J, Rajabi MJ. Advantage and drawback of support vector machine functionality. International Conference on Computer, Communications, and Control Technology (I4CT), IEEE 2014. p. 63-5.

[12] Koza JR. Genetic programming: on the programming of computers by means of natural selection: MIT press; 1992.

[13] John H. Holland, Adaptation in Natural and Artificial Systems: An Introductory Analysis with Applications to Biology, Control and Artificial Intelligence. MIT Press, Cambridge, MA; 1992.

[14] Kiani B, Gandomi AH, Sajedi S, Liang RY. New Formulation of Compressive Strength of Preformed-Foam Cellular Concrete: An Evolutionary Approach. Journal of Materials in Civil Engineering. 2016:04016092.

[15] Babanajad SK, Gandomi AH, Mohammadzadeh D, Alavi AH. Numerical modeling of concrete strength under multiaxial confinement pressures using linear genetic programming. Automation in Construction. 2013;36:136-44.

[16] Searson D, Willis M, Montague G. Co-evolution of non-linear PLS model components. Journal of Chemometrics. 2007;21:592-603.

[17] Searson DP, Leahy DE, Willis MJ. GPTIPS: an open source genetic programming toolbox for multigene symbolic regression. Proceedings of the International multiconference of engineers and computer scientists: Citeseer; 2010. p. 77-80.

[18] Hii C, Searson DP, Willis M. Evolving toxicity models using multigene symbolic regression and multiple objectives. Int J Mach Learn Comput. 2011;1:30-5.

[19] Searson DP. GPTIPS 2: an open-source software platform for symbolic data mining. arXiv preprint arXiv:14124690. 2014.

[20] Valencia-Ramirez JM, Raya J, Cedeno JR, Suarez RR, Escalante HJ, Graff M. Comparison between Genetic Programming and full model selection on classification problems. Power, Electronics and Computing (ROPEC), 2014 IEEE International Autumn Meeting on: IEEE; 2014. p. 1-6.

[21] Bažant ZP. Prediction of concrete creep and shrinkage: past, present and future. Nuclear Engineering and Design. 2001;203:27-38. 
[22] Bazant ZP, Li G-H. Comprehensive database on concrete creep and shrinkage. ACI Materials Journal. 2008;105:635-7.

[23] Rubin D. Multiple imputation for nonresponse in surveys. New York, NW: J Wiley and Sons, 1987.

[24] Shamshirband S, Khoshnevisan B, Yousefi M, Bolandnazar E, Anuar NB, Wahab AWA et al. A multi-objective evolutionary algorithm for energy management of agricultural systems - a case study in Iran. Renewable and Sustainable Energy Reviews. 2015;44:457-65.

[25] Deb K, Pratap A, Agarwal S, Meyarivan T. A fast and elitist multiobjective genetic algorithm: NSGA-II. Evolutionary Computation, IEEE Transactions on. 2002;6:182-97.

[26] Gani A, Siddiqa A, Shamshirband S, Hanum F. A survey on indexing techniques for big data: taxonomy and performance evaluation. Knowledge and Information Systems. 2016;46:241-84.

[27] Sajjadi S, Shamshirband S, Alizamir M, Yee L, Mansor Z, Manaf AA et al. Extreme learning machine for prediction of heat load in district heating systems. Energy and Buildings. 2016;122:222-7.

[28] Gandomi AH, Roke DA, Sett K. Genetic programming for moment capacity modeling of ferrocement members. Engineering Structures. 2013;57:169-76.

[29] Huo XS, Al-Omaishi N, Tadros MK. Creep, shrinkage, and modulus of elasticity of highperformance concrete. ACI Materials Journal. 2001;98.

[30] Babanajad S, Roozbahani A, Zahraie B, Shekarchi M. Selecting a Proper Repair System for Rehabilitation of Deteriorated Structures in Severe Environmental Conditions. Journal of Performance of Constructed Facilities. 2015:04015080.

[31] Champiri MD, Attar A, Hanifehzadeh M, Willam K, Gencturk B. Long-Term Performance of Dry Storage Structures. CONCREEP 10. p. 1593-602.

[32] Champiri MD, Mousavizadegan SH, Moodi F. A decision support system for diagnosis of distress cause and repair in marine concrete structures. Computers \& Concrete. 2012;9:99-118.

[33] Moradllo MK, Shekarchi M, Hoseini M. Time-dependent performance of concrete surface coatings in tidal zone of marine environment. Construction and Building Materials. 2012;30:198-205.

[34] Sajedi S, Huang Q. Probabilistic prediction model for average bond strength at steel-concrete interface considering corrosion effect. Engineering Structures. 2015;99:120-31.

[35] Sajedi S HQ, Miran SA. Reliability-Based Life-Cycle-Cost-Analysis Of Corroded Reinforced Concrete Substructures Considering Patch Repair. Nace Corrosion Risk Management Conference. Paper No. RISK16-8732, Houston, Tx2016.

[36] Rahmani T, Kiani B, Bakhshi M, Shekarchizadeh M. Application of different fibers to reduce plastic shrinkage cracking of concrete. 7th RILEM International Conference on Cracking in Pavements: Springer; 2012. p. 635-42.

[37] Committee ACI. ACI 209.2 R-08: Guide for Modeling and Calculating Shrinkage and Creep in Hardened Concrete. American Concrete Institute Committee; 2008.

[38] Bazant ZP, Baweja S. Creep and shrinkage prediction model for analysis and design of concrete structures: Model B3. ACI Special Publications. 2000;194:1-84.

[39] Rubin DB. Multiple imputations in sample surveys-a phenomenological Bayesian approach to nonresponse. Proceedings of the survey research methods section of the American Statistical Association: American Statistical Association; 1978. p. 20-34.

[40] Allison PD. Missing data: Sage publications; 2001. 
1 [41] Carpenter J, Kenward M. Multiple imputation and its application: John Wiley \& Sons; 2012.

2 [42] Committee ACI. ACI 363R-92: State-of-the-Art Report on High-Strength Concrete. American

3 Concrete Institute Committee; 1997.

4 [43] Committee ACI. 209R-92: Prediction of Creep, Shrinkage, and Temperature Effects in Concrete 5 Structures American Concrete Institute Committee; 2008.

6 [44] Gandomi AH, Alavi AH, Mirzahosseini MR, Nejad FM. Nonlinear genetic-based models for 7 prediction of flow number of asphalt mixtures. Journal of Materials in Civil Engineering. 2010.

8 [45] béton Fid. Structural Concrete: Textbook on Behaviour, Design and Performance: Updated 9 Knowledge of the CEB-FIP Model Code 1990: FIB-Féd. Int. du Béton; 1999.

10 [46] Gardner N, Lockman M. Design provisions for drying shrinkage and creep of normal-strength 11 concrete. ACI Materials Journal. 2001;98.

12 [47] Gandomi A, Roke D. Intelligent formulation of structural engineering systems. Seventh MIT 13 Conference on Computational Fluid and Solid Mechanics-Focus: Multiphysics \& Multiscale, 14 Massachusetts Institute of Technology, Cambridge, MA2013. 